\title{
Immunological and virological aspects of HIV cell-to-cell transfer Olivier Schwartz
}

Address: Virus \& Immunity Unit, Department of Virology, Institut Pasteur, Paris, 75015, France from Frontiers of Retrovirology: Complex retroviruses, retroelements and their hosts

Montpellier, France. 21-23 September 2009

Published: 24 September 2009

Retrovirology 2009, 6(Suppl 2):I16 doi:10.1 186/1742-4690-6-S2-II6

This abstract is available from: http://www.retrovirology.com/content/6/S2/II6

(C) 2009 Schwartz; licensee BioMed Central Ltd.

HIV-1 spread in lymphocytes occurs either by free viral particles, released by infected cells, or much more efficiently by direct cell-to-cell transfer through mechanisms that include formation of virological synapses (VS), filopodia and nanotubes.

We have quantified and characterized further these diverse modes of contact, by confocal and electron microscopy, flow-cytometry, and real-time imaging of fluorescent HIV. Viral transmission mainly occurs across VS, and through "polysynapses", a poorly described rosette-like structure formed between one infected cell and multiple adjacent recipients. Polysynapses are characterized by simultaneous HIV clustering and transfer at multiple membrane regions. Furthermore, HIV Gag proteins often adopt a ring-like supramolecular organisation at sites of intercellular contacts and colocalize with CD63 tetraspanin and raft components GM1, Thy-1 and CD59. The role of various cellular and viral proteins during HIV cell-to-cell transfer will be presented.

We are also studying how HIV-1 infected T cells are sensed by the innate immune system, and activate the production of type I-IFN and other cytokines by plasmacytoid DCs and other cell types. Cell-to-cell transfer likely represents an efficient means for the virus to propagate and to perturb communication between immune cells. 